\title{
Expansile cough impulse: a useful clinical sign for empyema necessitans
}

\author{
Sayantan Banerjee, ${ }_{1}^{1}$ Sagnik Biswas, ${ }^{2}$ Animesh Ray ${ }_{1}{ }^{2}$ Venkatesh Vaithiyam ${ }^{2}$
}

${ }^{1}$ Infectious Diseases, All India Institute of Medical Sciences New Delhi, New Delhi, New Delhi, India

${ }^{2}$ Department of Internal Medicine, All India Institute of Medical Sciences, New Delhi, Delhi, India

Correspondence to Dr Animesh Ray, doctoranimeshray@gmail.com

Accepted 8 March 2019

\section{DESCRIPTION}

A 26-year-old man from India with no known prior comorbidities presented with a swelling on the right side of the chest wall below the right breast which had been gradually increasing in size over the last 6 months prior to presentation (figure 1).

He had experienced high-grade fever for 10 days, about 3 weeks after noticing the swelling for the first time. There was no history of chest pain, chronic or recurrent cough, haemoptysis, significant loss of weight or trauma to the chest wall.

He denied any family history of tuberculosis. On probing, he was able to recall an exposure to his university hostel roommate, 4years ago in 2012, from whom he used to learn to play the flute, sharing the same instrument. The roommate had a history of chronic cough and fever and was later diagnosed to have multidrug resistant (MDR) tuberculosis that year (2012).

Our patient had a body mass index of $15.61 \mathrm{~kg} /$ $\mathrm{m}^{2}$, without any significant findings on general physical examination. Chest wall examination revealed a $7.5 \times 4.5 \mathrm{~cm}$ fluctuant swelling on the anterolateral aspect of the right side of the chest wall, without any overlying skin changes. The mass was mobile and had a cystic consistency. An expansile cough impulse was also present (video 1). There was a significant volume loss of the right hemithorax with reduced respiratory excursion and crowding of the ribs on the right side. Breath sounds were diminished in the lower right hemithorax with dullness to percussion. Chest X-ray revealed a right-sided pleural effusion and subsequent contrast-enhanced CT scan confirmed the presence of a pleural effusion and subcutaneous swelling along with an intrathoracic communicating channel (figure 2). The pus was aspirated and showed GeneXpert positivity for Mycobacterium tuberculosis with rifampicin resistance. Liquid culture also revealed isoniazid and rifampicin resistance. He was started on a regimen for MDR tuberculosis as per the WHO Shorter MDR TB regimen (with 4 months of ethambutol, pyrazinamide,

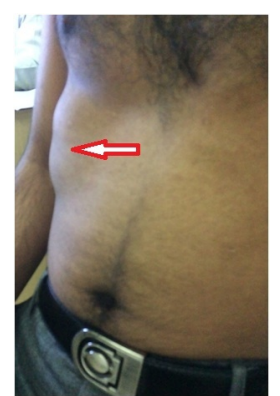

Figure 1 The swelling on the lower part of the right chest wall (marked by an arrow).

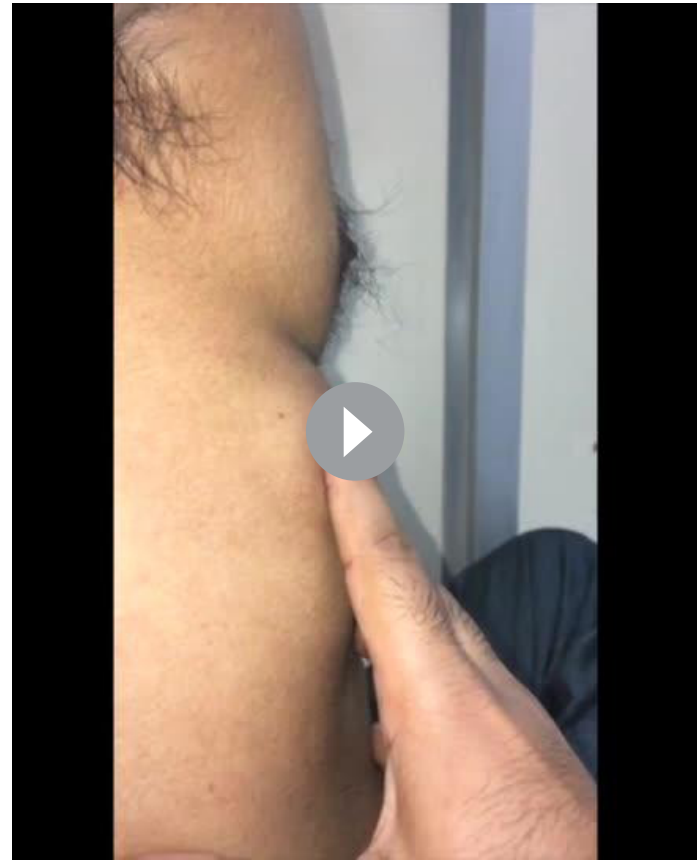

Video 1 Swelling seen on the anterior chest wall of the patient, along with expansile cough impulse (demonstrated).

moxifloxacin, kanamycin, ethionamide, high- dose isoniazid and clofazimine in the intensive phase followed by 5 months of continuation phase with ethambutol, pyrazinamide, moxifloxacin and clofazimine), and the swelling gradually resolved over a period of 4 months with therapy completed at 9 months. There was no recurrence of the swelling on follow-up.

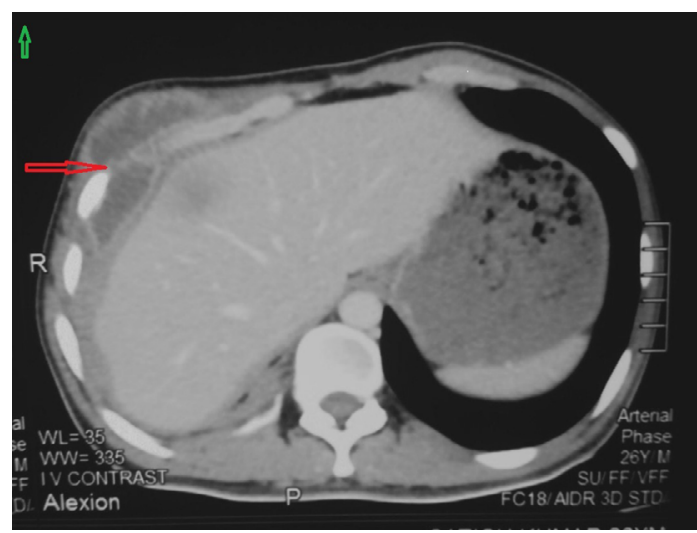

Figure 2 A contrast CT scan image showing the pleural collection, along with subcutaneous collection, with a connecting segment marked by an arrow. 
Empyema necessitans is an uncommonly encountered clinical presentation in the modern era due to early diagnosis and appropriate treatment. ${ }^{1}$ It is commonly caused by Mycobacterium tuberculosis, although other organisms like Staphylococcus aureus may cause similar presentations. ${ }^{2}$ It usually occurs on the anterior chest wall as the lung is more adherent to the posterior pleura. Besides empyema necessitans, it can also be encountered in lung herniation associated with trauma, where the presentation is acute. ${ }^{3}$ The pus so formed may even track down the anterior or posterior abdominal wall, where the mass, along with the presence of a cough impulse, may mimic an abdominal wall hernia. ${ }^{4}$ In our case, the expansile cough impulse was due to a communication between the pleural cavity and subcutaneous collection with transmission of positive intrathoracic pressure during coughing. A contrast-enhanced CT scan of the chest revealed findings that supported our proposed pathogenesis (figure 2).

\section{Patient's perspective}

I am a research scholar in neuroscience myself, but could not have thought that this mass could be due to an infection I might have contracted 4 years ago! I was very worried when I found an indrawing motion to the swelling when I was coughing because from whatever medical material I found online, it seemed to happen only when the lung is displaced from the chest wall. Tuberculosis may have decreased from the past, but it still lingers in society and occurs in unexpected ways. It can catch anyone unexpectedly!

\section{Learning points}

- Empyema necessitans is an uncommon complication of a pleural space infection, presenting as a chest wall or abdominal masses.

- A chest wall mass may present with expansile cough impulse on clinical examination, which might be indicative of the condition.

- Mycobacterium tuberculosis and Staphylococcus aureus are leading causes of empyema necessitans

Contributors $\mathrm{SBa}, \mathrm{SBi}, \mathrm{VV}$ and $\mathrm{AR}$ were involved in diagnosis and case management. The article was drafted by SBa, VV and SBi under the guidance of AR.

Funding The authors have not declared a specific grant for this research from any funding agency in the public, commercial or not-for-profit sectors.

Competing interests None declared.

Patient consent for publication Obtained.

Provenance and peer review Not commissioned; externally peer reviewed.

\section{REFERENCES}

1 Sharma V, Blyth KG. Empyema necessitans and a persistent air leak associated with rupture of an anaerobic lung abscess due to bacteroides. Thorax 2018;73:91-3.

2 Sindel EA. Empyema necessitates. Q Bull Sea View Hosp 1940;6:1-49.

3 Shankar S, Abbas A. Acute respiratory failure caused by spontaneous herniation of the lung. Ochsner J 2010;10:256-8.

4 Inada T, Ide H, Shiomi K, et al. [A case of tuberculous pyothorax with retroperitoneal gravitation abscess]. Nihon Kyobu Shikkan Gakkai Zasshi 1994;32:662-5.

Copyright 2019 BMJ Publishing Group. All rights reserved. For permission to reuse any of this content visit

https://www.bmj.com/company/products-services/rights-and-licensing/permissions/

BMJ Case Report Fellows may re-use this article for personal use and teaching without any further permission.

Become a Fellow of BMJ Case Reports today and you can:

- Submit as many cases as you like

- Enjoy fast sympathetic peer review and rapid publication of accepted articles

- Access all the published articles

- Re-use any of the published material for personal use and teaching without further permission

For information on Institutional Fellowships contact consortiasales@bmjgroup.com

Visit casereports.bmj.com for more articles like this and to become a Fellow 